\title{
Endosonographic Features of Advanced Gastric Carcinoma Invading the Colon: Is it Easy to Differentiate from Pancreatic Invasion?
}

E. A. Sabet, T. Okai, T. Minamoto, N. Sawabu

Dept. of Internal Medicine and Medical Oncology, Cancer Research Institute, Kanazawa University, Kanazawa, Japan

Corresponding Author

T. Okai, M.D.

Dept. of Internal Medicine and Medical Oncology

Cancer Research Institute

Kanazawa University

4-86 Yoneizumi

Kanazawa 921-8044

Japan

Fax: $\quad+81-76-226-2539$

E-mail: okai@kenroku.kanazawa-u.ac.jp

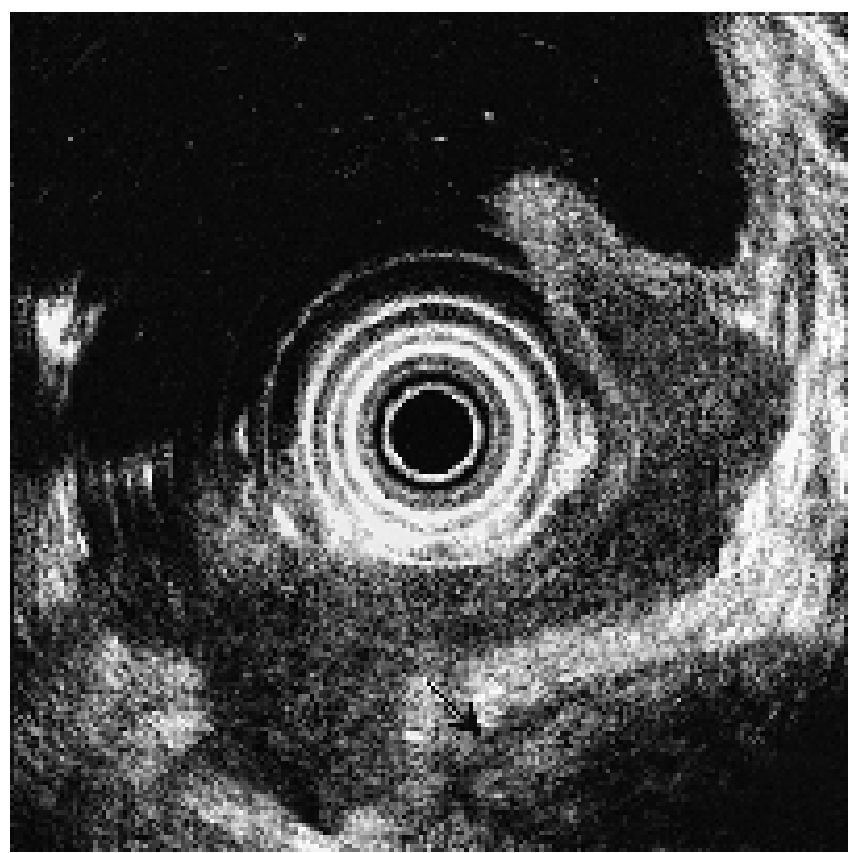

Endoscopic ultrasonographic (EUS) image of a T4 gastric carcinoma on the posterior wall of the angulus taken using a GF-UM200 (7.5 MHz) echoendoscope (Olympus, Tokyo, Japan). The linear hypoechoic area along the outer tumor margin (arrow) was first interpreted as the main pancreatic duct of an invaded pancreatic body, but surprisingly, the actual pancreas was demonstrated soon afterwards in its normal position.

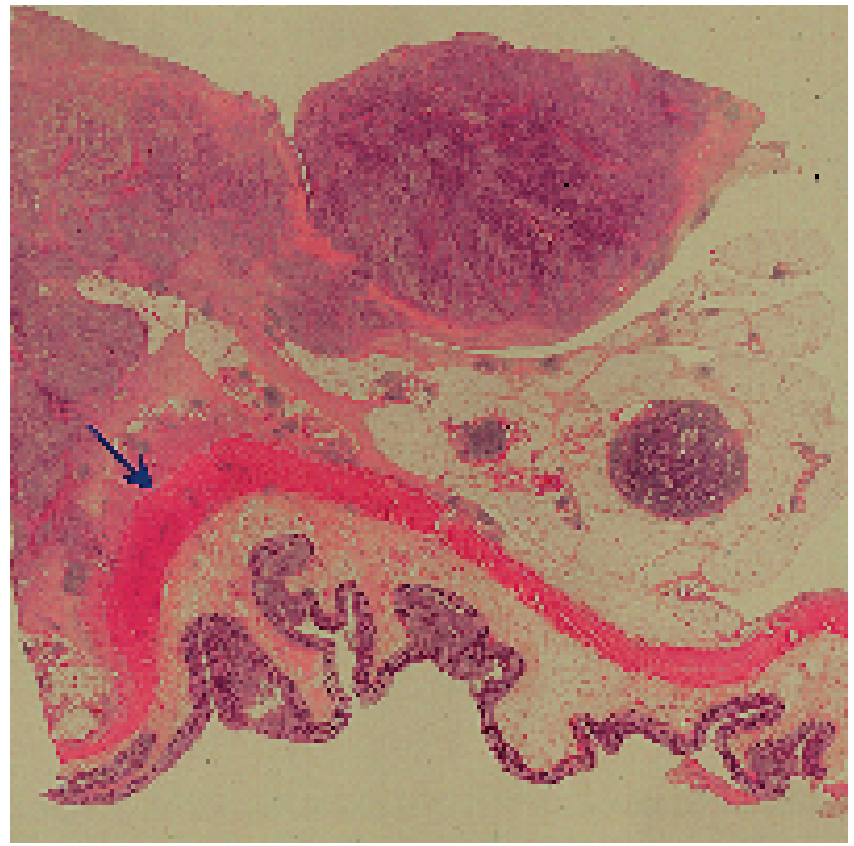

Histopathological examination proved that the linear hypoechoic area was consistent with the muscularis propria of the colon (arrow) (hematoxylin and eosin, original magnification $\times 5$ ). Another patient recently presenting with the same EUS features was diagnosed correctly. 